\title{
Medizinische Behandlung und Betreuung von Menschen mit Behinderung
}

\begin{abstract}
I. Präambel
Eine bedeutende Anzahl [1] von Menschen aller Lebensalter ist im Laufe des Lebens selber von Behinderung [2] betroffen. Ihre Stellung in der Gesellschaft wurde in den letzten Jahrzehnten vermehrt reflektiert und in den Kontext der allgemeinen Menschenrechte gestellt [3]. Menschen mit Behinderung sind in mehrfacher Weise bedroht: durch bevormundende Einschränkung der selbstbestimmten Lebensführung oder durch Vernachlässigung, durch gesellschaftliche Barrieren oder durch aktive Ausgrenzung. Zur Abwehr solcher Bedrohungen sind die Anerkennung und Unterstützung des Rechts auf Selbstbestimmung und Eigenverantwortung sowie die Beseitigung von Hindernissen für die freie Teilhabe am gesellschaftlichen Leben unerlässliche Voraussetzungen. Zudem ergibt sich die Notwendigkeit aktiver Schritte zum Einbezug von Menschen mit Behinderung in alle Gesellschaftsbereiche.

Behinderungen variieren in ihrer Art, in ihrem Schweregrad, in ihrer Dauer und in ihrem sozialen Kontext in einem ausserordentlich breiten, kontinuierlichen Spektrum. Ihre Bedeutung für die medizinische [4] Behandlung und Betreuung kann dementsprechend sehr unterschiedlich sein.

Eine Behinderung kann in direktem Zusammenhang mit der Behandlung stehen: Präventive, kurative, rehabilitative und palliative Massnahmen sollen die Auswirkungen der angeborenen oder erworbenen Beeinträchtigung beseitigen oder vermindern.
\end{abstract}

Sie kann die Behandlung und Betreuung aber auch nur indirekt beeinflussen: Auch wenn Gesundheitsstörungen in keinem direkten Zusammenhang mit einer Behinderung stehen, kann diese für den Verlauf der Krankheit oder die diagnostischen und therapeutischen Möglichkeiten wichtig sein. Es muss deshalb eine der besonderen Situation angepasste Vorgehensweise gewählt werden.

In vielen Fällen steht die Behinderung aber in keinem relevanten Zusammenhang zur Gesundheitsstörung, die behandelt werden muss. In diesen Situationen ist eine von der üblichen Therapie abweichende Behandlung ebensowenig gerechtfertigt, wie es eine Diskriminierung nach Geschlecht oder Nationalität wäre.
Ziel der Richtlinien ist es,

- den Anspruch aller Menschen mit Behinderung auf angemessene Behandlung und Betreuung zu bekräftigen;

- auf die entscheidende Rolle aufmerksam zu machen, die eine gute medizinische Behandlung und Betreuung für die Unterstützung von Menschen mit Behinderung in ihrem Streben nach Selbstbestimmung und sozialer Teilhabe spielt;

- Ärzten [5], Pflegenden und Therapeuten in den Richtlinien eine Hilfe zu bieten für den Umgang mit Menschen mit Behinderung und ihren Angehörigen

- und gesellschaftlichen Institutionen und politischen Instanzen Empfehlungen abzugeben, wie günstige Rahmenbedingungen für eine gute medizinische Behandlung und Betreuung von Menschen mit Behinderung errichtet werden können.

Sie schliessen damit die in den Richtlinien zur «Behandlung und Betreuung von älteren, pflegebedürftigen Menschen» bewusst offen gelassene Lücke der Behandlung und Betreuung jüngerer pflegebedürftiger Personen, sind aber auch für die Behandelnden und Betreuenden von Patienten mit Behinderung, die nicht pflegebedürftig sind, von Belang.

\section{Richtlinien}

\section{Geltungsbereich}

Die vorliegenden Richtlinien richten sich an Ärzte [6], Pflegende und Therapeuten, die Menschen mit Behinderung medizinisch behandeln und betreuen oder als Gutachter beurteilen [7], sei dies zu Hause oder in medizinischen, sozialmedizinischen oder sozialen [8] Institutionen.

«Behinderung» bezeichnet im folgenden die erschwerenden Auswirkungen eines angeborenen oder erworbenen Gesundheitsproblems [9] auf die alltäglichen Aktivitäten der betroffenen Person und auf ihre Teilhabe am gesellschaftlichen Leben. Die Behinderung resultiert aus dem Wechselspiel zwischen körperlicher Schädigung, funktioneller Beeinträchtigung [10] und sozialer Einschränkung der betroffenen Person sowie den 
behindernden oder fördernden Umständen und den Erwartungen ihres Lebensumfeldes. Ihre Ausprägung und ihr subjektives Erleben werden moduliert durch die Persönlichkeitsmerkmale des einzelnen Menschen mit Behinderung [11].

Die Richtlinien beschränken sich bewusst nicht auf bestimmte Behinderungsformen, sondern sollen in allen Fällen zur Anwendung kommen, in denen eine Behinderung den Behandlungs- und Betreuungsprozess massgeblich prägt. Einzelne Abschnitte dieser Richtlinien können demnach für die Betreuung von Menschen mit verschiedenen Behinderungsformen und -graden von unterschiedlicher Relevanz sein.

\section{Grundsätze}

Im folgenden werden die Grundwerte, Haltungen und Handlungsweisen hervorgehoben, von denen diese Richtlinien ausgehen.

\subsection{Achtung der Menschenwürde}

Die Würde [12] ist mit dem Menschsein gegeben, ist also unabhängig von den körperlichen, geistigen oder psychischen Beeinträchtigungen eines Menschen oder von einem bestimmten Kontext. In diesem Sinn muss die Würde bedingungslos respektiert werden. In Situationen der Schwäche ist die Gefahr, dass die Würde missachtet wird, besonders gross.

Die Würde des Patienten [13] zu achten bedeutet für die Behandelnden und Betreuenden,

- den Menschen mit Behinderung in seiner Einzigartigkeit zu sehen und ihm individuell als Mann oder Frau, gemäss seinem Alter und seiner Entwicklung zu begegnen;

- der besonderen Verletzbarkeit des Patienten sowohl im Verhalten als auch in der Kommunikation Rechnung zu tragen und ihm mit Respekt, Einfühlung und Geduld zu begegnen;

- den Patienten in seinen Bemühungen um ein selbstbestimmtes und integriertes Leben zu unterstützen;

- sich über bestehende Macht- und Abhängigkeitsverhältnisse Rechenschaft abzulegen;

- sich bewusst zu machen, dass Vorstellungen von Normalität und Anderssein, Partizipation und Ausgrenzung von der eigenen Perspektive abhängen.

\subsection{Respektierung der Autonomie}

Jede Person hat das Recht auf Selbstbestimmung. Dies gilt auch dann, wenn eine Behinderung zu einer Einschränkung der Autonomiefähigkeit führt. Autonomiefähig ist eine Person, die in der Lage ist, ihre Lebenssituation zu erfassen, daraus Entscheidungen abzuleiten, die in Übereinstimmung mit ihren Werten und Überzeugungen ste- hen, und ihren diesbezüglichen Willen auszudrücken.

Autonomie zu respektieren bedeutet für die Behandelnden und Betreuenden,

- die notwendigen Teilprozesse der Autonomiefähigkeit (Erfassen der Situation, Erkennen von unterschiedlichen Entscheidungsmöglichkeiten und ihren Konsequenzen, deren Wertung aufgrund der eigenen Präferenzen, Äusserung von getroffenen Entscheidungen und Wünschen sowie deren Umsetzung) ohne Druck und empathisch zu unterstützen;

- bei Patienten mit eingeschränkter Autonomiefähigkeit zu ermitteln, welche Teilfähigkeiten vorhanden sind und wie diese bei der Entscheidfindung berücksichtigt werden können;

- dem Patienten nahestehende Personen zur Unterstützung der Kommunikation einzubeziehen, soweit dies dem Wunsch bzw. dem Interesse des Patienten entspricht;

- für die Kommunikation genügend Zeit und die nötigen Hilfsmittel aufzuwenden;

- sich Rechenschaft darüber abzulegen, dass das notwendige Erspüren des Patientenwillens durch Empathie sowohl beim Betreuungsteam als auch bei den Angehörigen [14] die Gefahr der Projektion eigener Wünsche und Vorurteile mit sich bringt.

\subsection{Gerechtigkeit und Partizipation}

In einer am Grundsatz der Gerechtigkeit orientierten Gesellschaft hat die gleichberechtigte Teilhabe aller Mitglieder am gesellschaftlichen Leben einen hohen Stellenwert. Da eine Einschränkung der Partizipationsmöglichkeiten oft eine der wesentlichen Konsequenzen einer Schädigung oder Funktionseinbusse ist, stellt die Gesellschaft für Menschen mit Behinderung besondere Ressourcen zur Verfügung. Eine wichtige Komponente dieser gesellschaftlichen Leistung ist die medizinische Behandlung und Betreuung. Diese hat sich deshalb stets auch an den Auswirkungen ihrer Massnahmen auf die Partizipationsmöglichkeiten des Patienten zu orientieren. Als Unterstützung dafür dient die ICF-Klassifikation der WHO [15].

\subsection{Berücksichtigung der Lebensqualität} Die medizinische Behandlung und Betreuung von Patienten mit Behinderung ist immer auch im Hinblick auf ihre Auswirkungen auf die Lebensqualität zu beurteilen. Die Lebensqualität adäquat zu berücksichtigen, sei dies systematisch mittels eines validierten Instruments oder rein situationsbezogen, bedeutet für die Behandelnden und Betreuenden, 
- das subjektive Erleben des Patienten, insbesondere seine Zufriedenheit mit seiner Lebenssituation, in den Vordergrund zu stellen;

- die für die Fragestellung relevanten Dimensionen der Lebensqualität (physisch, emotional, intellektuell, spirituell, sozial und ökonomisch) zu erfassen;

- die Gewichtung der verschiedenen Aspekte der Lebensqualität aus der Sicht des Patienten zu achten;

- bei Patienten mit beeinträchtigten Kommunikationsmöglichkeiten das subjektive Erleben des Patienten soweit wie möglich aufgrund objektivierbarer Beobachtungen zu erschliessen;

- bei der Anwendung der Beobachtung als Zugang zum subjektiven Erleben des Patienten sich die Gefahr der Projektion eigener Vorurteile und Wünsche bewusst zu machen;

- die Lebensqualität von Angehörigen und Betreuenden, die oft eng verknüpft mit derjenigen des Patienten ist, explizit zu thematisieren und getrennt zu beurteilen.

Die Beurteilung medizinischer Massnahmen unter dem Aspekt der Lebensqualität wird erschwert, wenn der Patient die Auswirkungen nicht selbst abschätzen kann oder in seinen Kommunikationsmöglichkeiten eingeschränkt ist. Besonders heikel ist der Einsatz von medizinischen Massnahmen, die zwar längerfristig die Lebensqualität verbessern oder aufrechterhalten können, diese aber vorübergehend beeinträchtigen. Bei Patienten, die nicht selbst entscheiden können und deren mutmasslicher Wille nicht bekannt ist, hat die Abwägung gestützt auf die medizinethischen Prinzipien «Gutes tun» und «Nicht schaden» zu erfolgen.

\subsection{Fürsorge und Verantwortung}

Bei manchen Menschen mit schweren Behinderungen reichen die Gewährung der Selbstbestimmung und der Abbau von Schranken der Partizipation nicht aus, sie benötigen besondere Fürsorge durch betreuende Personen. Das Angewiesensein auf Fürsorge begründet Abhängigkeiten, die das Selbstwertgefühl der Betroffenen stören und sie verletzlich für Missbrauch durch andere machen können. Es ist wichtig, dass Betreuende und Betreute sich vor Augen halten, dass Abhängigkeiten zu den Grundbedingungen menschlichen Lebens gehören.

Die Fürsorgebedürftigkeit von Menschen mit Behinderung verlangt die Übernahme von Verantwortung durch Betreuende, sei es in der Familie, durch persönliche Assistenz [16] oder in sozia- len oder sozial-medizinischen Institutionen. Die Betreuung von Menschen mit Behinderung kann befriedigend, aber auch herausfordernd und belastend sein. Ein übersteigertes Verantwortungsgefühl verstärkt sowohl die Abhängigkeit der betreuten wie auch die Verletzbarkeit der betreuenden Person. Die Vorstellung, allein für das Wohl des betreuten Menschen verantwortlich zu sein, kann zum Raubbau an den eigenen Kräften bis zur Erschöpfung führen. Sie kann auch anfällig machen für Kränkungen durch die Umgebung, die scheinbar oder tatsächlich zu wenig Verständnis aufbringt. Wenn die betreute Person ihrerseits die wechselseitige Abhängigkeit vom Betreuer ausnützt, um inadäquate Forderungen zu stellen, kann dadurch die Situation zusätzlich kompliziert werden.

In der medizinischen Betreuung ist auf Abhängigkeiten von Menschen mit Behinderung sowie auf eine besondere Verletzbarkeit von Betreuenden zu achten und ihnen situationsgerecht Rechnung zu tragen.

\subsection{Angemessene medizinische Behandlung und Betreuung}

Jeder Mensch hat Anspruch auf eine angemessene medizinische Behandlung und Betreuung. Dies bedeutet einerseits, dass keine indizierte präventive, kurative oder palliative Massnahme aufgrund einer bestehenden Schädigung oder Beeinträchtigung vorenthalten werden darf. Jede Diskriminierung im Zugang zu Leistungen des Gesundheitswesens ist zu vermeiden. Andererseits erfordert eine angemessene Behandlung und Betreuung auch, dass die Auswirkungen der vorbestehenden Schädigung auf den möglichen Therapieerfolg berücksichtigt und die individuellen Wünsche und Bedürfnisse des Patienten gebührend beachtet werden. Ausschlaggebendes Kriterium für die Angemessenheit einer Massnahme ist ihr positiver Einfluss auf Lebensqualität und Lebensdauer.

\subsection{Persönliche, kontinuierliche Betreuung und interdisziplinäre Zusammenarbeit}

Menschen mit Behinderung haben oft vielfältige medizinische Probleme und werden deshalb häufig von Spezialisten unterschiedlicher Fachrichtungen betreut. Regelmässige Aufenthaltswechsel zwischen Institution und Familie können zur gleichzeitigen oder sequentiellen Behandlung durch verschiedene Ärzte führen. Aus diesem Grund muss ein für den Patienten primär zuständiger Arzt, bei dem alle relevanten Informationen zusammenfliessen, bezeichnet sein. Bei einem Wechsel der Zuständigkeit, z.B. beim Über- 
gang ins Erwachsenenalter, muss eine sorgfältige Übergabe stattfinden, am besten in einem direkten Gespräch beider Ärzte mit dem Patienten.

Die Pflege und im medizinischen Umfeld angebotene nichtärztliche Therapien sind in die beschriebene persönliche und kontinuierliche Betreuung einzubeziehen. Es muss für die gegenseitige Information und Koordination aller involvierten Ärzte, Pflegenden und Therapeuten gesorgt werden, sofern der Patient damit einverstanden ist. Oft sind auch Angehörige sozialer und pädagogischer Berufe einzubeziehen. Die interdisziplinäre Koordination kann fallbezogen geschehen, z.B. in sogenannten Helferkonferenzen bei Krisensituationen. Für die Langzeitbetreuung spezifischer Behinderungsformen sind spezialisierte, interdisziplinäre, ambulante Sprechstunden die optimale Lösung.

Für Patienten mit kognitiver oder psychischer Beeinträchtigung kann der Kontakt mit vielen verschiedenen Ärzten verwirrend und beängstigend sein. Dadurch werden sie in ihrer ohnehin eingeschränkten Autonomiefähigkeit zusätzlich beeinträchtigt. Eine Vertrauensperson, die mit der Krankengeschichte des Patienten gut bekannt ist, soll ihn deshalb zu allen Arztbesuchen begleiten dürfen.

\subsection{Zusammenarbeit mit dem sozialen Umfeld}

Bei der Behandlung und Betreuung von Patienten mit Behinderung in sozialen und sozial-medizinischen Institutionen oder im Akutspital sollen der behandelnde Arzt und die Ansprechperson der Pflege guten Kontakt zu den nahen Bezugspersonen des Patienten unterhalten. Angehörige von pflegebedürftigen Patienten mit Behinderung haben bei deren Betreuung meist grosse Verantwortung übernommen; ihre Erfahrung in der Interpretation von Symptomen und ihre intime Kenntnis von Gewohnheiten, Vorlieben und Abneigungen des Patienten sind deshalb als wichtige Entscheidungshilfen stets ernst zu nehmen.

Die Betreuung von pflegebedürftigen Patienten mit Behinderung zuhause führt oft zu grossen Belastungen. Ärzte, Pflegende und Therapeuten haben die Aufgabe, die Angehörigen oder andere betreuende Personen zu beraten und zu unterstützen.

\section{Kommunikation}

Eine offene, adäquate und einfühlsame Kommunikation mit dem Patienten ist Voraussetzung und Bestandteil jeder guten Behandlung und Betreuung. Nicht alle Patienten mit Behinderung benötigen eine Unterstützung oder spezielle
Kommunikationsformen. Diese Patienten in ihren Fähigkeiten zu unterschätzen kann kränkend sein.

Für Patienten mit spezifischen Bedürfnissen muss die Kommunikationsweise der jeweiligen Situation angepasst werden. Es ist darauf zu achten, dass

- das Gespräch wenn möglich an einem dem Patienten bekannten Ort mit vertrauten Gesprächspartnern stattfindet;

- wegen einer Seh- oder Hörbehinderung benötigte Hilfsmittel optimal eingesetzt werden und die Umgebungsbedingungen (Lichtverhältnisse, Hintergrundlärm) für ein Gespräch günstig sind;

- die das Gespräch führende Medizinalperson sich mit dem intellektuellen Niveau, auf dem mit dem Patienten kommuniziert werden kann, vertraut gemacht hat und mit allenfalls benötigten Kommunikationshilfsmitteln (Piktogramme, Zeigetafeln, Computer usw.) umgehen kann;

- allenfalls benötigte Drittpersonen wie Dolmetscher, z.B. für die Gebärdensprache, bzw. Angehörige oder enge Betreuungspersonen, die die nichtsprachlichen Zeichen des Patienten gut verstehen oder sogar über ein eigenes Kommunikationssystem mit ihm verfügen, anwesend sind;

- von Anfang an der unter Umständen vermehrte Zeitbedarf des Menschen mit Behinderung zur Aufnahme, Verarbeitung und Vermittlung von Informationen eingeplant wird; - der Patient auch bei erschwerter Kommunikation immer direkt angesprochen und auf keinen Fall über seinen Kopf hinweg diskutiert und entschieden wird.

\subsection{Information über die medizinische Behandlung und Betreuung}

Eine ausreichende und verständliche Information über alle vorgesehenen ärztlichen, pflegerischen oder therapeutischen Massnahmen ist notwendig, damit Patienten ihr Recht auf Selbstbestimmung wahrnehmen können. Durch eine Behinderung kann die Möglichkeit, routinemässig vermittelte Informationen zu verstehen oder sich aktiv um zusätzliche Erläuterungen und Kenntnisse zu bemühen, eingeschränkt sein. Insbesondere Patienten mit kognitiver oder psychischer Beeinträchtigung oder mit Kommunikationsbeeinträchtigungen sind in hohem Grade abhängig von der Art und Weise, wie sie informiert werden.

Das Verständnis der dargebotenen Information und die Entscheidfindung können erleichtert und dem Patienten Sicherheit und Orientie- 
rung vermittelt werden, wenn der medizinische Gesprächspartner:

- auf eine einfache Sprache achtet (kurze Sätze, Vermeiden von Fremdwörtern, Abkürzungen und unterschiedlichen Bezeichnungen für gleiche Begriffe);

- langsam und deutlich und mit Blickkontakt auf Augenhöhe spricht;

- die Information in kleine Portionen aufteilt;

- Aussagen in Variationen wiederholt und mit Gesten, Bildern, Piktogrammen usw. unterstützt;

- dem Patienten geplante medizinische Massnahmen durch das Vorführen von Abläufen, das Zeigen von Instrumenten und den Besuch von Örtlichkeiten näherbringt.

Der Einbezug von Drittpersonen (Angehörigen, gesetzlichen Vertretern, Vertrauenspersonen) richtet sich nach den Regeln in Kapitel 4. Dabei ist $\mathrm{zu}$ beachten, dass diese in unterschiedlichen Rollen auftreten können:

- Drittpersonen können unverzichtbare Hilfen in der Kommunikation zur Ermittlung des Patientenwillens leisten;

- Drittpersonen können sich gemäss den Regeln in Kapitel 4 an stellvertretenden Entscheiden bei urteilsunfähigen Patienten beteiligen;

- Drittpersonen können auch als Vertreter ihrer eigenen Interessen auftreten, die sich nicht unbedingt mit dem Patienteninteresse dekken müssen.

Diese Rollen sind unter Umständen schwer auseinanderzuhalten. In vielen Fällen ist es deshalb wichtig, auch ein Gespräch mit dem Patienten allein zu führen.

\subsection{Information über Diagnose und Prognose}

Eine für alle sehr schwierige Situation ist die Information des Patienten oder seiner Angehörigen über eine Diagnose, die zu einer Behinderung führt oder führen könnte. Dies kann eine einmalige Aufgabe sein oder auch im Verlaufe fortschreitender Leiden mehrmals notwendig werden. Es braucht besonderes Einfühlungsvermögen, um ein realistisches Bild der Beeinträchtigung und ihrer Folgen zu vermitteln, ohne alle Hoffnungen zu zerstören. Dazu muss genügend Zeit zur Verfügung stehen, damit die betroffenen Personen die Nachricht verarbeiten und erste Fragen stellen können. Das erste Gespräch sollte nicht mit Ratschlägen überfrachtet sein, da die intellektuelle Aufnahmefähigkeit oft durch Emotionen beeinträchtigt ist. Die betroffenen Perso- nen sollen in verständlicher Form über die Beeinträchtigung, ihre Auswirkungen und allfällige Therapien aufgeklärt werden. Sie sollen insbesondere auch auf existierende Selbst- und Fachhilfen bzw. Informationsmöglichkeiten und die Möglichkeit psychologischer Unterstützung hingewiesen werden. Besondere Aufmerksamkeit ist auf die bei solchen Gesprächen bewusst oder unbewusst vermittelten nichtverbalen Botschaften $\mathrm{zu}$ richten, da diese den Betroffenen sehr nahegehen. Oft bleibt lebenslang unvergessen, ob der Gesprächspartner Abwertung, Abgrenzung und Hoffnungslosigkeit oder Wertschätzung, Empathie und Zuversicht gezeigt hat.

\section{Entscheidungsprozesse [17]}

Entscheidungsverfahren und Strukturen müssen die spezifische Situation des Patienten berücksichtigen. Insbesondere soll darauf geachtet werden, dass der Patient und seine allfälligen Vertreter ausreichend und verständlich informiert werden [18] und Entscheidungen ohne Druck fällen können.

\subsection{Urteilsfähige Patienten}

Ärzte, Pflegende und Therapeuten dürfen eine Behandlung oder Betreuung nur mit der freien Einwilligung des urteilsfähigen [19], informierten Patienten durchführen.

Auch Minderjährige und entmündigte Erwachsene können in bezug auf ihre Angelegenheiten urteilsfähig sein und über eine medizinische Behandlung und Betreuung entscheiden. Im Vorfeld einer solchen Entscheidung muss die Urteilsfähigkeit sorgfältig abgeklärt werden.

Es ist Pflicht der Fachleute, Minderjährige oder entmündigte Erwachsene soweit als möglich in Entscheidungen einzubeziehen und ihre $\mathrm{Zu}$ stimmung zu erhalten. Sie sollen beim Entscheid nicht überfordert werden; es soll aber auch nicht über sie entschieden werden, wenn sie willens und fähig sind, Entscheidungen über ihre Behandlung und Betreuung aktiv mitzutragen. Bei Entscheidungen von grosser Tragweite sollen die gesetzlichen Vertreter (Eltern, Vormund) einbezogen werden, sofern dies der Patient nicht ablehnt.

Ist ein minderjähriger oder ein entmündigter Patient mit einer vorgeschlagenen Behandlung und Betreuung, die er in ihrer Bedeutung und Tragweite versteht, nicht einverstanden, darf diese nicht durchgeführt werden [20]. Dabei ist jedoch sorgfältig zu prüfen, ob die Verweigerung wirklich seinem freien Willen entspricht [21].

Bei manchen Patienten (insbesondere bei einer psychischen Beeinträchtigung) kann die 
Urteilsfähigkeit im Laufe der Zeit wechselhaft ausgeprägt sein. Patientenverfügungen sowie Hinweise auf den mutmasslichen Willen des Patienten sind deshalb von besonderer Bedeutung.

\subsection{Nichturteilsfähige Patienten [22]}

Entscheidungen über die Behandlung und Betreuung von Patienten, die nie urteilsfähig waren [23], sollen sich ausschliesslich an deren wohlverstandenen Interessen orientieren und im Konsens mit den gesetzlichen Vertretern [24] getroffen werden. Der nichturteilsfähige Patient soll dennoch so weit als möglich in die Entscheidfindung mit einbezogen werden.

War der Patient zu einem früheren Zeitpunkt urteilsfähig, soll sich die Behandlung und Betreuung an seinem mutmasslichen Willen orientieren. Im Vordergrund stehen hierbei frühere schriftliche Äusserungen des Patienten selbst (Patientenverfügung) oder Aussagen der vom Patienten eingesetzten Vertretungsperson in medizinischen Angelegenheiten (Vertrauensperson) oder dem Patienten nahestehender Personen. Hat der Patient keinen Vertreter und keine Angehörigen [25], die über seinen mutmasslichen Willen Auskunft geben können, ist insbesondere bei länger dauernder Behandlung oder risikoreichen Eingriffen die Vormundschaftsbehörde zu informieren.

Verweigern die gesetzlichen Vertreter ihre Zustimmung zu einer im Interesse des Patienten liegenden Behandlung, ist die Vormundschaftsbehörde zu informieren. Reicht die Zeit zur Information der Vormundschaftsbehörde infolge Dringlichkeit [26] des Eingriffs nicht aus, soll und darf der Eingriff auch gegen den ausdrücklich erklärten Willen des Vertreters vorgenommen werden.

\subsection{Entscheidungsfindung im Betreuungsteam}

Medizinische Behandlungen oder Betreuungsmassnahmen erfordern oft einen interdisziplinären Entscheidungsprozess. Bevor einem Patienten solche Behandlungen oder Massnahmen vorgeschlagen werden, sollen sie im Betreuungsteam diskutiert werden. Dabei soll ein Entscheid angestrebt werden, der von allen Beteiligten mitgetragen werden kann. Wesentliche Beschlüsse werden schriftlich festgehalten, regelmässig überprüft und allenfalls angepasst [27].

Die Notwendigkeit der interdisziplinären Zusammenarbeit entbindet die behandelnden Ärzte, Pflegenden und Therapeuten nicht von ihrer Verantwortung für die Entscheidungen und Massnahmen in ihrem Zuständigkeitsbereich.

\section{Medizinische Behandlung und Betreuung}

\section{1 Ätiologische Diagnostik}

Menschen mit Behinderung haben ein Anrecht auf eine angemessene Diagnostik zur Erforschung der Natur und Ursache ihres Gesundheitsproblems. Eine möglichst präzise Abklärung der Ursachen einer Schädigung bzw. Beeinträchtigung kann dazu beitragen, dass die Betroffenen, ihre Angehörigen sowie die behandelnden und betreuenden Medizinalpersonen

- präventive, kurative und rehabilitative Behandlungspläne zur direkten Beeinflussung der Beeinträchtigung spezifischer gestalten;

- aufgrund der Ursache typischerweise zu erwartende Komplikationen und Zusatzerkrankungen besser verhüten, erkennen und behandeln;

- die Tatsache der Beeinträchtigung besser in die Lebensgeschichte einordnen und verarbeiten können;

- genetische Risiken im Hinblick auf die Familienplanung kennen und berücksichtigen.

\subsection{Gesundheitsförderung und Prävention}

Es ist darauf zu achten, dass Menschen mit Behinderung von allen Massnahmen der Gesundheitsförderung und Prävention profitieren können, die der Allgemeinbevölkerung angeboten werden. Impfungen und Vorsorgeuntersuchungen sind in den dafür vorgesehenen Altersabschnitten oder Risikosituationen zu empfehlen und bei Einverständnis durchzuführen.

Das der Behinderung zugrundeliegende Gesundheitsproblem sowie die daraus resultierenden Lebensumstände können die Risikosituation im Vergleich zu Personen der Allgemeinbevölkerung verändern. So muss beispielsweise mit Risiken aufgrund von Bewegungsmangel, Fehlernährung, Selbstwertproblemen, Ängsten, Bedrohung durch Übergriffe oder Vernachlässigung vermehrt gerechnet werden. Betroffene und ihre Betreuer sollen in geeigneter Form über vorliegende Risiken aufgeklärt und über Möglichkeiten der Gesundheitsförderung informiert werden.

Für viele genetische Störungen, Fehlbildungen oder chronische Krankheiten, die bei Patienten mit Behinderung vorkommen, haben medizinische Fachgesellschaften spezifische Vorsorgeprogramme aufgestellt und publiziert. Die darin empfohlenen regelmässigen Kontrolluntersuchungen erlauben eine optimale Früherkennung und Therapie von Komplikationen und Zusatzerkrankungen. Es gehört zur medizinischen Behandlung und Betreuung von Menschen mit Behinderung, ihnen den Zugang zu diesen Leistungen zu ermöglichen. 


\subsection{Akuttherapie}

Das Recht auf unverzügliche medizinische Hilfe bei akuter Erkrankung oder Verletzung durch angemessene Diagnostik und wirksame Therapie gilt für alle Menschen unabhängig von Art und Ausmass einer Behinderung. Behinderungsspezifische Besonderheiten können die Anamneseerhebung, die Untersuchung, die Interpretation von Befunden und die Wahl und Applikation der bestmöglichen Therapie erschweren. Das der Behinderung zugrundeliegende Gesundheitsproblem oder die besondere Lebensweise können das Ansprechen auf Standardtherapien, insbesondere mit Medikamenten, verändern und unerwartete Nebenwirkungen auslösen. Dem soll einerseits durch den Beizug von Angehörigen, Betreuern und früheren behandelnden Medizinalpersonen, die den Patienten besser kennen, andererseits durch den Erwerb behinderungsspezifischer Kenntnisse aus der Literatur oder durch den konsiliarischen Beizug von Experten begegnet werden.

Die Komplexität des Problems, die vor Ort verfügbaren Kompetenzen, die zeitliche Dringlichkeit und die geographischen Verhältnisse entscheiden darüber, ob eine Abklärung und Behandlung am Ort der Erstversorgung möglich ist oder ob eine Verlegung in ein spezialisiertes Zentrum angestrebt werden soll. Dabei ist auch dem speziellen Pflegebedarf Rechnung zu tragen, der notwendig ist, um das bestehende Ausmass der Selbständigkeit aufrechtzuerhalten. Betreuungspersonen aus dem familiären oder institutionellen Umfeld sollen im Spital beratend und unterstützend, jedoch nicht als Ersatz für kompetentes Pflegepersonal zugezogen werden.

Nach Überwinden der Akutsituation muss frühzeitig abgeklärt werden, ob eine rasche Rückkehr in die früheren Lebensumstände möglich ist oder ob vorübergehend oder dauernd andere Lösungen gefunden werden müssen. Auch hierfür kann der Zuzug von Experten oder die Überweisung in eine spezialisierte Institution sinnvoll sein.

\subsection{Behandlung von psychischen Störungen}

Psychische Erkrankungen können zu einer vorübergehenden oder dauernden Beeinträchtigung führen oder eine Behinderungssituation komplizieren.

Psychische Störungen können reaktiv auf eine körperliche oder geistige Beeinträchtigung bzw. die durch diese bedingten Lebensumstände entstehen, jedoch auch ohne erkennbaren $\mathrm{Zu}$ sammenhang zur vorbestehenden Behinderung auftreten.
Oft bedarf es eines spezialisierten psychiatrischen Wissens, um psychische Störungen von Verhaltensauffälligkeiten abzugrenzen, die aufgrund von Kommunikationsstörungen oder ungeeigneten Lebensbedingungen resultieren. Ebenso braucht es spezielle Kenntnisse, um eine Diagnose zu stellen und die Behandlung durchzuführen. Insbesondere sind Probleme der Kommunikation und der Kognition aufgrund einer Behinderung zu erkennen und ist ein adäquates therapeutisches Setting zusammen mit dem Patienten und seinen Angehörigen und Betreuenden aufzubauen. Psychoaktive Medikamente müssen nach fachlichen Kriterien und nicht mit dem primären Ziel, den Betreuungsaufwand zu reduzieren, eingesetzt werden. Sie sind bezüglich Wirkung und Nebenwirkungen im weiteren Verlauf sorgfältig zu überprüfen.

Die oft über Jahre belastende Situation der Angehörigen und Betreuenden ist gebührend zu beachten, und es ist ihnen nötigenfalls Hilfe zu vermitteln.

\subsection{Rehabilitation}

Rehabilitation nach einer Krankheit oder einem Unfall bzw. Habilitation nach angeborener oder frühkindlicher Schädigung umfasst alle medizinischen Massnahmen, die die Auswirkungen nicht heilbarer Folgeschäden so weit als möglich vermindern, und hat deshalb für Menschen mit Behinderung ganz besondere Bedeutung. Körperliche, psychische und soziale Fähigkeiten und Möglichkeiten sollen möglichst weitgehend wiedererlangt, entwickelt und erhalten werden. Das Rehabilitationsteam mit spezifischer Schulung und Erfahrung und der Patient mit seinen persönlichen Lebensvorstellungen und -wünschen müssen gemeinsam das Rehabilitationspotential abschätzen und individuelle Rehabilitationsziele erarbeiten.

Rehabilitation ist grundsätzlich langfristig angelegt und braucht deshalb genügend Zeit. Bei bestimmten Gesundheitsproblemen ist eine gezielte und intensive Rehabilitation nötig, die das Ausmass der Schädigung vermindern kann. Gleichzeitig muss aber der fortlaufenden Entwicklung Rechnung getragen werden. Diese kann durch Fortschreiten oder Besserung der zugrundeliegenden Krankheit, durch neu auftretende zusätzliche Erkrankungen, durch das zunehmende Alter sowie durch psychische und soziale Umbrüche oder schleichende Veränderungen gekennzeichnet sein. Es braucht deshalb lebenslänglich regelmässige Standortbestimmungen mit einer standardisierten Evaluation der biopsychosozialen Situation. Daraus entstehen neue, angepasste Rehabilitationsziele, die eine wirk- 
same und bedürfnisorientierte Weitertherapie ermöglichen.

\subsection{Palliative Care [28]}

Angeborene oder erworbene Schädigungen oder Beeinträchtigungen können so schwer sein, dass von Anfang an oder infolge fortschreitender Verschlechterung eine verminderte Lebenserwartung ohne Heilungsaussicht besteht. Begleitend zu den rehabilitativen Anstrengungen ist deshalb ein ausreichendes Angebot von Massnahmen bereitzustellen, die die körperlichen Symptome lindern und gleichzeitig die sozialen, seelischgeistigen und religiös-spirituellen Bedürfnisse der Betroffenen berücksichtigen.

Das Erkennen und Erfassen von körperlichen Symptomen wie Schmerz, Muskelverspannung, Übelkeit und Atemnot einerseits und negativen Emotionen wie Angst, Einsamkeit, Wut und Trauer andererseits ist bei Patienten mit eingeschränkter Kommunikationsfähigkeit sehr anspruchsvoll und erschwert eine angemessene Palliative Care. Die Kenntnis und Anwendung geeigneter Assessmentinstrumente sind in der Betreuung dieser Patienten unabdingbar.

Bei der Interpretation neu aufgetretener oder sich verschlimmernder Symptome ist stets sorgfältig abzuklären, ob es sich um eine nichtbeeinflussbare Verschlechterung der Grundkrankheit oder um eine neu erworbene, behandelbare Gesundheitsstörung handelt. Es ist darauf zu achten, dass nicht durch eine einseitige Fokussierung auf die symptomatische Behandlung sinnvolle kurative Optionen übergangen werden. Mögliche diagnostische und therapeutische Schritte müssen in einer sorgfältigen ethischen Entscheidungsfindung bezüglich Nutzen und Belastung für den Patienten evaluiert werden. Begleitend sind in jedem Fall palliative Massnahmen einzuleiten.

\subsection{Sterben und Tod [29]}

Tritt eine unabwendbare Verschlechterung des Gesundheitszustandes ein, sind bevorstehendes Sterben und der nahe Tod mit dem Patienten und seinen Angehörigen in geeigneter Form zu thematisieren. Die Art und Weise, wie die Behinderung das Leben des Patienten und seine Kommunikation mit den ärztlichen, pflegerischen und therapeutischen Bezugspersonen geprägt hat, kann auch für den Dialog in dieser letzten Lebensphase wegleitend sein. Mitunter kann es sinnvoll sein, mögliche Optionen am Lebensende und das Abfassen einer Patientenverfügung bereits zu einem früheren Zeitpunkt anzusprechen.

Auf die schwierige emotionale Situation langjähriger Betreuungspersonen in Familie oder Institution ist Rücksicht zu nehmen, indem sie in die Gestaltung der Sterbephase in angemessener Weise miteinbezogen und nach Bedarf unterstützt werden.

\section{Dokumentation und Datenschutz}

\subsection{Krankengeschichte und Pflege- dokumentation}

Über jeden Patienten wird eine fortlaufende Patientendokumentation geführt, die auf aktuellem Stand gehalten wird [30]. Diese sollte neben den Angaben betreffend Anamnese, Diagnose und Krankheitsverlauf, angeordnete Behandlungen usw. insbesondere auch den Ablauf und Gegenstand der Aufklärung des Patienten bzw. seines gesetzlichen Vertreters dokumentieren. Bei älteren Jugendlichen und entmündigten Erwachsenen sollte das Patientendossier zudem begründete Aussagen darüber enthalten, für welche Entscheide der Patient als nicht urteilsfähig betrachtet wurde. Werden freiheitsbeschränkende Massnahmen eingesetzt, so sind diese ebenfalls im Patientendossier festzuhalten. Die Dokumentation soll Angaben über die Gründe, die Art der angewendeten Massnahme, deren Zweck und Dauer sowie über die Ergebnisse der regelmässigen Neubeurteilung beinhalten.

\subsection{Vertraulichkeit und Auskunftspflichten gegenüber Dritten}

Der Arzt, das Pflegepersonal und die Therapeuten sind an die Schweigepflicht gebunden [31]. Die Erhebung, die Ablage, die Auswertung und die Weitergabe von Daten dürfen nur unter Beachtung der gesetzlichen Datenschutzbestimmungen erfolgen. Auskünfte an Dritte dürfen nur mit ausdrücklicher Zustimmung des Patienten bzw. seiner Vertreter oder aufgrund einer gesetzlichen Grundlage oder aufgrund einer schriftlichen Bewilligung der vorgesetzten Behörde oder Aufsichtsbehörde erteilt werden [32].

\section{Zwangsmassnahmen [33]}

Infolge psychischer Krankheit oder aus anderen Gründen bei Patienten mit Behinderung auftretende Verhaltensstörungen, Unruhe oder Verwirrtheit können zu einer Gefährdung ihrer selbst oder anderer und/oder einer schweren Störung des Gemeinschaftslebens führen. Die Anwendung von Zwangsmassnahmen [34] zur Vermeidung solcher Gefährdungen stellt einen Eingriff in die Grundrechte dar und muss deshalb grundsätzlich die Ausnahme bleiben. Sie muss verhältnismässig sein und sich an den Interessen des Patienten orientieren. Eidgenössische und kantonale Vorschriften sind zu beachten. 


\subsection{Bedingungen [35]}

Eine Zwangsmassnahme darf nur eingesetzt werden, wenn alle nachfolgenden Bedingungen erfüllt sind:

- das Verhalten des Patienten gefährdet in erheblichem Masse sein Leben, seine Gesundheit oder diejenige anderer Personen oder stellt eine schwerwiegende Störung des Gemeinschaftslebens dar;

- das beobachtete auffällige Verhalten ist nicht auf behebbare Ursachen zurückzuführen wie Schmerz, Nebenwirkungen von Medikamenten oder störende Einflüsse des Umfeldes wie z.B. zwischenmenschliche Spannungen bei den Betreuenden, ungenügende Kommunikation, ungeeignete Wohn- oder Betreuungsverhältnisse;

- andere, die persönliche Freiheit weniger beeinträchtigende Massnahmen können nicht zielführend angewendet werden.

Auch für die Durchführung von Zwangsmassnahmen besteht eine Aufklärungspflicht bzw. das Erfordernis der Einwilligung des Patienten oder seines Vertreters. Die Anwendung soll vom Arzt und den betreuenden Personen gemeinsam besprochen werden, bevor sie dem Patienten oder seinem Vertreter vorgeschlagen wird. Ist der Patient urteilsunfähig und hat weder Vertrauensperson noch gesetzlichen Vertreter, ist die Vormundschaftsbehörde möglichst rasch über die Durchführung von Zwangsmassnahmen zu informieren.

Verweigern der urteilsfähige Patient oder die gesetzlichen Vertreter die Zustimmung zu einer Zwangsmassnahme, ist die Vormundschaftsbehörde zu informieren. Reicht die Zeit zur Information der Vormundschaftsbehörde infolge Dringlichkeit nicht aus, soll und darf eine Massnahme auch gegen den ausdrücklich erklärten Willen des Patienten oder des Vertreters vorgenommen werden. Von einer einzigen Fachperson einer Institution in einer Notfallsituation gefällte kurzfristige Entscheide sollen anschliessend im Team überprüft werden.

\subsection{Begleitmassnahmen}

Stets sollte bewusst sein, dass bei Zwangsmassnahmen die Gefahr von Schädigungen besteht. Deshalb muss während der Dauer der Massnahme die bestmögliche Überwachung sichergestellt sein. Die Massnahme wird in regelmässigen Abständen evaluiert [36]; die Häufigkeit richtet sich nach der Art der Massnahme. Die Massnahme wird beendet, sobald die Bedingungen gemäss Kapitel 7.1 nicht mehr erfüllt sind.

\section{Misshandlung und Vernachlässigung}

\subsection{Definitionen}

Körperliche, psychische und sexuelle Übergriffe und Misshandlungen sowie Vernachlässigung sind Ausdruck des Missbrauchs eines Abhängigkeitsverhältnisses. Das Recht schützt die Integrität der Person und betrachtet deshalb Übergriffe und Misshandlungen als Straftaten [37].

Menschen, die sich aufgrund körperlicher, kognitiver oder psychischer Beeinträchtigungen weniger gut zur Wehr setzen können, werden häufiger Opfer von Übergriffen und Misshandlungen. Eine besondere Abhängigkeitssituation, wie sie sich in sozialen Institutionen oder im ärztlichen, pflegerischen oder therapeutischen Behandlungsverhältnis findet, wirkt ebenfalls als ein die Gefahr verstärkender Faktor. Die besondere Verletzbarkeit des Opfers und die Stärke des Abhängigkeitsverhältnisses begünstigen dabei nicht nur das Auftreten von Übergriffen, sondern erschweren auch deren Aufdeckung.

Vernachlässigung bedeutet die ungenügende Erfüllung einer Fürsorgepflicht, die einer Betreuungsperson aufgrund eines gesetzlichen oder freiwillig eingegangenen Verantwortlichkeitsverhältnisses zur betreuten Person auferlegt ist. Die Fürsorgepflicht besteht darin, die auf Fürsorge angewiesene Person in der Erfüllung ihrer Bedürfnisse zu unterstützen, soweit diese nicht selbst dazu in der Lage ist. Von Vernachlässigung können alle Stufen der Bedürfnispyramide (existentielle Bedürfnisse, Sicherheitsbedürfnis, Bedürfnis nach Zugehörigkeit, nach Achtung und Wertschätzung und nach Selbstverwirklichung) [38] betroffen sein. Dabei kann paradoxerweise eine Vernachlässigung des Bedürfnisses nach Selbstverwirklichung auch durch übermässige Fürsorge entstehen, die eine betreute Person an ihrer Entfaltung und Autonomieentwicklung hindert.

Ursachen für Vernachlässigung können ungenügende materielle Ressourcen, ungünstige Umgebungsbedingungen, fehlende Zeit und ungenügende Kenntnisse und Erfahrung der Betreuungspersonen sowie eine gestörte Beziehung zur betreuten Person sein.

\subsection{Erkennen von Risikosituationen und Prävention}

Ärzte, Pflegende und Therapeuten sind verpflichtet, an die Gefahr von Übergriffen, Misshandlungen und Vernachlässigungen zu denken und entsprechende Risikosituationen zu erkennen und zu vermeiden. Dies gilt sowohl für die Betreuung in medizinischen, sozial-medizinischen und sozialen Institutionen als auch im familiären Um- 
feld. Das Augenmerk ist insbesondere auf folgende Situationen zu richten:

- Situationen mit hohem Betreuungsaufwand bei verhältnismässig niedrigen Betreuungsressourcen;

- Überforderungssituationen bei Betreuungspersonen in Ausbildung oder mit noch geringer Erfahrung;

- Betreuungspersonen mit Überlastungssymptomen oder auffälligem Beziehungsverhalten;

- die Anwendung von Zwangsmassnahmen;

- die Durchsetzung von Regeln des Zusammenlebens;

- die Gefahr, dass Menschen mit Behinderung durch Veränderungen im Gesundheitszustand oder in der Betreuungssituation in eine Krisensituation geraten und besonders verletzbar werden können.

Allein schon die regelmässige Thematisierung der Möglichkeit von Übergriffen, Misshandlungen und Vernachlässigungen sowie die bewusste Pflege eines respektvollen und wertschätzenden Umgangs mit betreuten Personen kann präventive Wirkung entfalten. Kritische Alltagssituationen in medizinischen, sozial-medizinischen und sozialen Institutionen wie die Unterstützung von Körperpflege, Essen, Ausscheidung und ZuBett-Gehen sowie medizinische Untersuchungen oder die Anwendung therapeutischer Massnahmen sollen auf ihr Gefahrenpotential für Übergriffe hin angeschaut und durch geeignete Regeln und Unterstützungsmöglichkeiten abgesichert werden.

Auch bei Betreuungssituationen in der Familie ist die Sensibilität für Risikosituationen wichtig. Potentiell kritische Situationen sollen angesprochen und ein allfälliger Bedarf nach Unterstützung abgeklärt und erfüllt werden.

\subsection{Vorgehen bei konkretem Verdacht}

Falls ein konkreter Verdacht auf Übergriffe, Misshandlung oder Vernachlässigung besteht, muss in erster Linie die betroffene Person geschützt und vor weiterem Schaden bewahrt werden. Ein solcher kann sowohl aus der Fortdauer der festgestellten Situation wie auch aus unsachgemässen Aufklärungsmassnahmen und Interventionen entstehen.

In medizinischen, sozial-medizinischen und sozialen Institutionen sind Verdachtsfälle einer besonders erfahrenen, unabhängigen Fachperson zur Erfassung und weiteren Behandlung zu melden. Auch bei der Feststellung eines Misshandlungsverdachts bei Familienbetreuung ist es ratsam, nicht als Einzelperson aktiv zu werden, sondern den Rat von unabhängigen Fachpersonen einzuholen. Dabei sind die ärztliche Schweigepflicht und allfällige kantonale Bestimmungen bezüglich Melderechte und -pflichten [39] zu beachten. In jedem Fall ist für eine psychologische Betreuung des Opfers zu sorgen.

\section{Sexualität}

Die Sexualität ist ein integraler Bestandteil der Persönlichkeit. Die sexuelle Selbstbestimmung muss geschützt werden. Eine Behinderung kann auf körperlicher, geistiger oder psychischer Ebene die Entfaltung der Sexualität beeinträchtigen, indem sie Partnersuche, Gestaltung sexueller Beziehungen und den Umgang mit Konsequenzen und Risiken sexueller Aktivität erschwert. Dabei spielen neben der Behinderung meist auch die durch diese bedingten Lebensumstände eine wichtige Rolle.

Ärzten, Pflegenden und Therapeuten kann die Aufgabe zukommen, Menschen mit Behinderung im Umgang mit medizinischen Aspekten ihrer sexuellen Aktivität und deren Konsequenzen zu beraten und zu unterstützen. Insbesondere bei körperlichen Beeinträchtigungen ist es wichtig, den Unterstützungsbedarf in diesem Bereich aktiv anzusprechen, da er vom Patienten oft aus Scham verschwiegen wird. Insbesondere bei kognitiven Beeinträchtigungen ist die Sexualaufklärung eine wichtige Fördermassnahme für die sexuelle Gesundheit.

Bei der Beratung ist davon auszugehen, dass Menschen mit Behinderung das Recht auf ungehinderte Entfaltung ihrer Sexualität haben. Eine Begrenzung sexueller Aktivität ist nur dort statthaft, wo sie selbst oder andere Personen zu Schaden kommen oder öffentliches Ärgernis erregt wird.

Bei Menschen mit eingeschränkter Urteilsfähigkeit ist besondere Sorgfalt angebracht. Sie brauchen Unterstützung in der Entfaltung ihrer Sexualität und in der Überwindung entsprechender Hindernisse. Es darf dabei aber nicht zu einer einseitigen Förderung sexueller Aktivität kommen, mit der die Entfaltung der Persönlichkeit nicht Schritt halten kann.

\subsection{Konsequenzen und Risiken sexueller Aktivität}

Sexuelle Aktivität, soweit sie sich nicht auf die eigene Person beschränkt, lässt Bindungen zum Partner und zu allenfalls gezeugten Kindern entstehen und fordert damit die Übernahme von Verantwortung. Sie birgt aber auch Risiken, so das Risiko sexuell übertragener Infektionen oder das Risiko des Missbrauchs durch den Partner. Behinderungen können sowohl die Übernahme 
von Verantwortung als auch den Umgang mit Risiken erschweren.

Es ist ärztliche Aufgabe, für sexuell aktive Menschen mit Behinderung die Beratung und Unterstützung sowohl bezüglich Antikonzeption, Kinderwunsch oder unerwünschter Schwangerschaft als auch bezüglich der Verhütung sexuell übertragener Infektionen und der Vorbeugung des sexuellen Missbrauchs sicherzustellen. Bei sexuell aktiven Frauen ist an die gynäkologische Vorsorgeuntersuchung zu denken.

\subsection{Antikonzeption und Sterilisation}

Aus der Vielzahl von Verhütungsmitteln gilt es für Menschen mit Behinderung die individuell am besten geeignete Methode auszuwählen. Reversiblen Verhütungsmitteln ist immer der Vorzug zu geben vor einer definitiven Massnahme, wie sie eine Sterilisation darstellt.

Bei der Wahl der Methode ist zu beachten, dass diese

- mit allenfalls vorhandenen körperlichen Gesundheitsproblemen und damit einhergehenden Medikationen verträglich ist;

- eine möglichst selbständige und unkomplizierte Anwendung durch die betroffene Person selbst erlaubt oder das Umfeld eine allenfalls nötige Unterstützung gewährleisten kann;

- in ihrer Anwendung und ihren Nebenwirkungen den individuellen Vorstellungen und Bedürfnissen der betroffenen Person entspricht und ihre Akzeptanz findet.

Die Unterbindung ist im Sterilisationsgesetz [40] geregelt. Eine Unterbindung ist ein schwerer Eingriff in die körperliche und psychische Integrität und darf grundsätzlich nur bei Urteilsfähigen mit deren freier Einwilligung nach entsprechender Aufklärung durchgeführt werden.

Damit kommt der Feststellung der Urteilsfähigkeit eine zentrale Funktion zu. Nach Gesetz müssen Ärzte, die den Eingriff durchführen, dokumentieren, aufgrund welcher Feststellungen sie die betreffende Person als urteilsfähig erachten. Bei Menschen, deren Urteilsfähigkeit infolge einer kognitiven oder psychischen Beeinträchtigung nicht zweifelsfrei feststeht, ist eine Zweitmeinung einzuholen.

Das Gesetz verbietet die Sterilisation dauernd urteilsunfähiger Personen. Es sieht aber eine Ausnahme vor bei urteilsunfähigen Personen, die mindestens 16 Jahre alt sind, wenn der Eingriff nach den gesamten Umständen im Interesse der betroffenen Person liegt, eine andere Antikonzeption nicht möglich ist und die Zeugung und Geburt eines Kindes und dessen Trennung von der betroffenen Person nach Geburt wahrscheinlich sind.

Aus ärztlicher Sicht ist die Umsetzung dieser Gesetzesbestimmung sehr schwierig. Insbesondere die vorurteilsfreie Feststellung des Interesses der betroffenen Person, die den Zweck des Eingriffes und die Implikationen einer Schwangerschaft oder Elternschaft nicht verstehen kann, ist kaum lösbar. Dessen Abgrenzung von den Interessen der Betreuenden wirft zusätzliche Probleme auf. Jedenfalls ist zu beachten, dass Frauen, die nicht urteilsfähig sind, nicht nur Schutz vor einer unerwünschten Schwangerschaft, sondern auch vor sexuellem Missbrauch benötigen, denn das Wissen um die Sterilisation einer Frau mit einer kognitiven oder psychischen Beeinträchtigung kann das Missbrauchsrisiko erhöhen, weil keine Schwangerschaft zu befürchten ist.

In der Praxis sollte die Sterilisation bei einer dauernd urteilsunfähigen Person nicht zur Anwendung kommen müssen, da der für den Schutz einer betroffenen urteilsunfähigen Person vor sexuellem Missbrauch und der Ansteckung durch sexuell übertragene Infektionen notwendige Aufwand auch eine wirksame Schwangerschaftsverhütung ohne derart weitreichenden Eingriff in die körperliche Integrität erlauben sollte.

\subsection{Elternschaft}

Bei Kinderwunsch von Personen, deren Fähigkeit, selbständig für das Wohl ihrer Kinder zu sorgen, aufgrund ihrer Beeinträchtigung fraglich oder nicht vorhanden erscheint, soll versucht werden, durch beratende Begleitung einen freiwilligen Verzicht und eine optimale Antikonzeption zu erreichen. Genauso gründlich sollen alle Möglichkeiten der Unterstützung einer Elternschaft durch das Umfeld abgeklärt und gefördert werden.

Einer Behinderung zugrundeliegende Gesundheitsprobleme können sowohl die Fortpflanzungsfähigkeit beeinträchtigen als auch ein erhöhtes Wiederholungsrisiko für Nachkommen aufweisen. Es gehört zur ärztlichen Betreuung, in dieser Situation die Möglichkeit einer genetischen Beratung anzubieten, in der über das bestehende Risiko aufgeklärt wird, Handlungsalternativen aufgezeigt werden und über pränatale Diagnostik informiert wird.

\subsection{Schutz vor sexuellem Missbrauch}

Der sexuelle Missbrauch von Menschen mit Behinderung ist ein oft tabuisiertes Thema mit wahrscheinlich hoher Dunkelziffer. Vor allem Menschen mit kognitiver Beeinträchtigung werden häufiger Opfer sexueller Übergriffe als andere Bevölkerungsgruppen. Möglicherweise sind aber auch Menschen mit bestimmten motorischen, 
sensorischen oder psychischen Beeinträchtigungen vermehrt betroffen. Dabei stammen die Täter oft aus dem sozialen Nahbereich des Opfers.

Eine verständliche Aufklärung und geeignete Sexualerziehung stellen wichtige Mittel in der Prävention von sexuellem Missbrauch dar. Menschen mit kognitiver und kommunikativer Beeinträchtigung müssen lernen können, über ihre Erfahrungen mit erwünschter und unerwünschter Intimität zu sprechen und die Selbstbestimmung über ihren Körper wahrzunehmen. In medizinischen, sozial-medizinischen und sozialen Institutionen sind Risiken für sexuellen Missbrauch zu thematisieren und präventive Konzepte einzuführen.

Besteht ein konkreter Verdacht auf einen einmaligen oder wiederholten sexuellen Missbrauch, ist sinngemäss wie in Kapitel 8.3 beschrieben vorzugehen. Zusätzliche ärztliche Aufgabe ist es, die allenfalls akut notwendige Infektionsprophylaxe sowie Antikonzeption zu vermitteln.

\section{Lebensabschnitte und Übergänge}

In der Behandlung und Betreuung von Patienten mit Behinderung ist, genau wie bei allen anderen Patienten, der Bedeutung der lebenslangen Entwicklung Rechnung zu tragen. Behinderung steht in einer Wechselwirkung mit Reife- und Alterungsprozessen. Auf der einen Seite können in jedem Alter neue Kompetenzen erworben werden, die zur selbstbestimmten Lebensgestaltung beitragen. Auf der anderen Seite können Fähigkeiten durch Krankheit oder höheres Alter eingeschränkt werden oder verloren gehen, was zu grösserer Abhängigkeit führen kann.

Je nach Art der Behinderung kann eine Diskrepanz zwischen dem entsprechend dem Lebensalter zu erwartenden und dem tatsächlichen Verhalten bestehen. Die Bedürfnisse solcher Patienten in bezug auf Betreuung, insbesondere bezüglich einer respektvollen Art der Kommunikation [41], müssen sorgfältig abgeklärt und die Behandlung angepasst werden.

\section{1 Übergang vom Kindes- ins Erwachsenenalter}

Der Übergang ins Erwachsenenalter ist durch verschiedene Veränderungen gekennzeichnet. Diese können den Alltag stark prägen und für den betroffenen Menschen Gewinn bringen, aber auch mit Gefühlen von Verlust einhergehen. Dies betrifft unter anderem:

- Veränderungen in langfristigen konstanten Beziehungen: insbesondere Eltern, aber auch Schul- und Heimkollegen;

- Veränderungen der Wohnsituation;
- vormundschaftliche Massnahmen;

- die zunehmende Bedeutung von Sexualität;

- das Eingehen einer Partnerschaft;

- den Austritt aus der Schule;

- den Eintritt ins Berufsleben oder die Aufnahme einer anderen Beschäftigungsform;

- den Vergleich mit Geschwistern in bezug auf Beruf, Partnerschaft usw.;

- den Übergang von der Kinder- und Jugendmedizin zur Erwachsenenmedizin;

- die Änderung der finanziellen Träger [42].

Die Entlassung aus einer pädagogischen Beziehung soll individuell angepasst erfolgen und eine Beziehung unter gleichberechtigten Erwachsenen ermöglichen. Dies bedeutet beispielsweise, dass Jugendliche schrittweise in Entscheidungen einbezogen werden und der Einbezug der Eltern sorgfältig abgeklärt wird und differenziert erfolgt. Starre Altersgrenzen sind in der medizinischen Betreuung hinderlich [43].

Auch für Jugendliche mit kognitiver Beeinträchtigung ist die Entwicklung einer weiblichen oder männlichen Geschlechtsidentität und -rolle wesentlich und in der Betreuung zu berücksichtigen. Die für die Übergangskrisen der Pubertät normalen Verhaltensänderungen sind abzugrenzen von Verhaltensauffälligkeiten, die im Zusammenhang mit der Beeinträchtigung stehen oder Ausdruck einer psychischen Störung sind.

\section{2 Übergang ins höhere Alter [44]}

Der Übergang ins höhere Alter ist durch verschiedene Veränderungen geprägt, die auch Gefühle der Unsicherheit und Trauer auslösen können. Dazu zählen insbesondere:

- Veränderungen im Beziehungsumfeld, insbesondere auch die Konfrontation mit Pflegebedürftigkeit und Tod der Eltern;

- Veränderungen der Wohnsituation, wie beispielsweise der Übertritt in ein Pflegeheim;

- der Verlust der Arbeit;

- altersbedingte körperliche und psychische Veränderungen;

- Änderung der finanziellen Träger [45].

Für die medizinische Betreuung und Behandlung sind deshalb die nachfolgenden Aspekte besonders zu beachten:

- Ältere Menschen mit Behinderung haben Anspruch auf die übliche Gesundheitsvorsorge, medizinische Diagnostik und Therapie. Symptome sollen diagnostisch geklärt und gezielt behandelt werden;

- bei gewissen Beeinträchtigungen treten altersspezifische Erkrankungen wie z.B. Demenz früher oder in atypischer Weise auf; 
- Sterben und Tod sollen in geeigneter Form thematisiert werden. Dabei ist auf die Möglichkeit des Erstellens einer Patientenverfügung hinzuweisen.

\section{3 Übertritt in eine soziale} oder sozial-medizinische Institution

Der Wechsel vom familiären Umfeld oder einer selbständigen Wohnform in eine soziale oder sozial-medizinische Institution bedeutet für jeden Menschen mit Behinderung eine einschneidende Veränderung. Meist sind mit dem Übertritt Änderungen des sozialen Umfelds und Auswirkungen auf die Möglichkeiten der Selbstbestimmung verbunden. Immer aber begibt sich der betroffene Mensch in die Obhut eines professionell organisierten und Autorität beanspruchenden Umfelds.

Im Zusammenhang mit der medizinischen Betreuung und Pflege verdienen nachfolgende Punkte besondere Beachtung:

- Vor jedem Übertritt in ein Pflegeheim, sei es aus dem familiären Umfeld oder sei es aus einer Behinderteninstitution, sind die ambulanten Möglichkeiten (z.B. Spitex) auszuschöpfen;

- der behandelnde Arzt ist verantwortlich für die Abklärung vor einem Übertritt, wie weit das pflegerische Wissen und die notwendigen Fertigkeiten zur Betreuung des Patienten beim Personal der in Frage kommenden sozialen Institution vorhanden sind. Er soll hierbei Rücksprache mit den Personen nehmen, die den Patienten bis jetzt betreut haben;

- zu einem geordneten Übertritt in eine soziale oder sozial-medizinische Institution gehört auch die Übergabe aller relevanten Informationen. Im Einverständnis mit dem Patienten oder seinem gesetzlichen Vertreter sollen pflegerische und biographische Informationen dem Betreuungsteam der Institution, ärztliche Unterlagen dem weiterbehandelnden Arzt übergeben werden;

- Menschen mit Behinderung bzw. deren gesetzliche Vertreter sind von der Institutionsleitung über das Recht der freien Arztwahl zu informieren. Diesbezüglichen Wünschen ist von seiten der Institution soweit möglich nachzukommen. Allfällige Einschränkungen der Arztwahl sind bei Eintritt zu besprechen.

Bei der Beantwortung der Frage, zu welchem Zeitpunkt der Wechsel von einer Behinderteninstitution in ein Pflegeheim zu erfolgen hat, ist von denselben Grundsätzen auszugehen wie bei Menschen ohne vorbestehende Beeinträchtigung.

\section{Forschung}

Menschen mit Behinderung dürfen nicht allein aufgrund ihrer Behinderung von der Teilnahme an Forschungsprojekten ausgeschlossen werden.

Menschen mit Behinderung leiden häufig an Störungen oder Krankheiten, über die wenig bekannt ist und für die keine oder nur beschränkt adäquate Therapiemöglichkeiten bestehen. Daten fehlen teilweise auch über die Interaktion von gesundheitlichen Störungen oder Krankheiten mit einer Behinderung. Aus diesem Grund besteht im Hinblick auf eine behinderungsspezifische Prävention, Therapie und Rehabilitation Forschungsbedarf und entsprechende Projekte sollten gezielt gefördert werden. Dabei müssen besondere Schutzbestimmungen beachtet werden. Insbesondere sollte diese Forschung mit urteilsunfähigen Kindern oder Erwachsenen mit Behinderung nur durchgeführt werden, wenn das Projekt nicht mit urteilsfähigen Personen durchgeführt werden kann. Eine Ablehnung der betroffenen Person, an einer Studie mitzuwirken, die von der Ablehnung einer medizinisch notwendigen Behandlung unterschieden werden kann, muss in jedem Fall respektiert werden [46].

\section{Empfehlungen}

Damit die für die Umsetzung der vorliegenden Richtlinien unerlässlichen Rahmenbedingungen erfüllt werden können, sind nachfolgende Empfehlungen massgebend.

\section{An politische Instanzen und Kostenträger}

- Berücksichtigung des erhöhten Bedarfs an personellen und baulichen Ressourcen für Menschen mit Behinderung bei der Planung und Realisierung von medizinischen Einrichtungen;

- Berücksichtigung der erhöhten Bedürfnisse von Menschen mit Behinderung bei der Gestaltung von Abgeltungssystemen für medizinische Leistungen;

- Garantie der Finanzierung für die Abklärung der Ursache von Behinderungen;

- Garantie für die Finanzierung von Langzeittherapien in der für die Erhaltung der Lebensqualität notwendigen Intensität; dies gilt auch, wenn kein Eingliederungsnutzen erwartet werden kann;

- Bereitstellung von Ressourcen in sozialen und sozial-medizinischen Institutionen für Notfalleintritte aus medizinischen Gründen oder infolge eines Zusammenbruchs des Betreuungssystems;

- Unterstützung der Betreuung und Pflege von Menschen mit Behinderung durch Angehörige; 
- Förderung der niederschwelligen und kompetenten Sexualberatung für Menschen mit Behinderung;

- Förderung von Modellen der unterstützten Elternschaft für Menschen mit Behinderung;

- Förderung von Projekten, welche Kinder von Eltern mit Behinderung unterstützen;

- Förderung von und Zusammenarbeit mit gemeinnützigen Organisationen der Fach- und Selbsthilfe für Menschen mit Behinderung.

\section{An Institutionen des Gesundheitswesens und der Medizin}

- Förderung der Aus-, Weiter- und Fortbildung der ärztlichen, pflegerischen und therapeutischen Mitarbeiter in behinderungsspezifischen Belangen, insbesondere auch in der Anwendung der ICF-Klassifikation der WHO und in der Feststellung der Urteilsfähigkeit bei Patienten mit eingeschränkten kognitiven, kommunikativen oder emotionalen Fähigkeiten;

- Förderung von Kompetenzzentren, interdisziplinären Spezialsprechstunden und Spezialistennetzwerken für besondere Behinderungsformen;

- Förderung der Erarbeitung und Verbreitung spezifischer Erkenntnisse zur Diagnostik und Therapie somatischer und psychischer Erkrankungen und zur Langzeitbetreuung von Menschen mit geistiger Behinderung;

- Partnerschaftliche Zusammenarbeit und Informationsaustausch mit dem Personal sozialer Institutionen;

- Förderung der Forschung im Bereich behinderungsspezifischer Prävention, Therapie und Rehabilitation;

- Förderung der Entwicklung und des Einsatzes von Hilfsmitteln für die unterstützte Kommunikation in allen Institutionen;

- Verzicht auf starre Altersgrenzen bei der Behandlung von Menschen mit Behinderung;

- Erstellen von institutionsinternen Richtlinien für das Vorgehen bei Verdacht auf Misshandlung und sexuelle Übergriffe (inkl. Umgang mit Schweigepflicht);

- Beachtung einer besonderen Sorgfaltspflicht bei der Anstellung von neuen Mitarbeitenden: z.B. Ansprechen des Themas «Übergriffe», Einholen von Referenzen, Verlangen eines Auszugs aus dem Zentralstrafregister.

\section{An soziale und sozial-medizinische Institutionen zur Betreuung von Menschen mit Behinderung}

- Sicherstellung der medizinischen Fachkompetenz;
- Unterstützung der betreuten Personen bezüglich medizinischer Therapien und Antikonzeption im Rahmen des Alltags;

- Regelung der Schnittstellen zur medizinischen Versorgung unter Berücksichtigung der Entscheidungsrechte gesetzlicher Vertreter und der Mitsprachebedürfnisse von Angehörigen;

- Bereitschaft für Notfalleintritte aus medizinischen Gründen oder infolge eines Zusammenbruchs des Betreuungssystems;

- Gewährleistung des Wissenstransfers aus der Institution zu den behandelnden Stellen der Akutmedizin;

- Dokumentation und Aufbewahrung biographischer und pflegerischer Daten;

- Ermöglichung der Begleitung von Patienten mit Behinderung durch eine Betreuungsperson der Institution zu Arztkonsultationen und Spitaleintritten;

- Unterstützung in der Abfassung von Patientenverfügungen, sofern gewünscht.

\section{Glossar}

\section{Behinderung}

«Behinderung» bezeichnet die erschwerenden Auswirkungen eines angeborenen oder erworbenen Gesundheitsproblems auf die alltäglichen Aktivitäten der betroffenen Person und auf ihre gleichberechtigte Teilhabe am gesellschaftlichen Leben. Die Behinderung resultiert aus dem Wechselspiel zwischen körperlicher Schädigung, funktioneller Beeinträchtigung und sozialer Einschränkung der betroffenen Person sowie den behindernden oder fördernden Umständen und den Erwartungen ihres Lebensumfeldes. Ihre Ausprägung und ihr subjektives Erleben werden moduliert durch die Persönlichkeitsmerkmale des einzelnen Menschen mit Behinderung.

Der Begriff wird in den vorliegenden Richtlinien auf der Basis der ICF-Klassifikation der WHO (Internationale Klassifikation der Funktionsfähigkeit, Behinderung und Gesundheit, Stand Oktober 2005) definiert. Diese dient als länder- und fachübergreifende einheitliche Sprache zur Beschreibung des funktionalen Gesundheitszustandes, der Behinderung, der sozialen Beeinträchtigung und der relevanten Umgebungsfaktoren einer Person. Eine kostenlose deutschsprachige Übersetzung kann unter www.dimdi.de/static/de/klassi/icf/index.htm heruntergeladen werden.

\section{Gesundheitsproblem}

«Gesundheitsproblem» ist der in der deutschen Übersetzung verwendete Begriff für das von der 
WHO eingeführte englische «health condition» (Definition: «Health conditions include diseases or illnesses, other health problems that may be short or long lasting, injuries, mental or emotional problems, and problems with alcohol or drugs», WHO Disability Assessment Schedule WHODAS II, http://whqlibdoc.who.int/publi cations/2000/a80933.pdf, Zugriff 31.8.2007; auf Deutsch: «Gesundheitsprobleme umfassen Krankheiten oder Befindlichkeitsstörungen, andere Gesundheitsstörungen von kurzer oder langer Dauer, Verletzungen, mentale oder emotionale Probleme sowie Probleme mit Alkohol und Drogen»).

\section{Menschenwürde}

Das ethische Konzept der Achtung der Würde geht von der universellen Intuition aus, dass jeder einzelne Mensch, weil er Mensch ist, in seiner Würde geachtet werden soll. Dies kommt darin zum Ausdruck, dass jedem Menschen einerseits besonderer Lebensschutz und Fürsorge, andererseits Achtung der Selbstbestimmung zustehen. In diesem Sinne darf der Begriff nicht mit einem Würdeverständnis verwechselt werden, das zur Beschreibung bestimmter Verhaltensweisen, besonderer Ausprägungen des sozialen Status oder bestimmter Fähigkeiten eines Menschen dient.

\section{Soziale Institution}

Als «soziale Institution» werden Institutionen bezeichnet, in denen Menschen mit Behinderung Bildung, Arbeit, Beschäftigung oder Betreuung erhalten. Damit sind nicht nur Heime, sondern auch andere Angebotsstrukturen (z.B. betreute Wohngruppen) gemeint.

\footnotetext{
Hinweise zur Ausarbeitung dieser Richtlinien

Auftrag: Am 18. Oktober 2005 hat die Zentrale Ethikkommission der SAMW eine Subkommission mit der Ausarbeitung von Richtlinien zur Behandlung und Betreuung von Menschen mit Behinderung beauftragt.

Verantwortliche Subkommission: Prof. Dr. med. Christian Kind, St. Gallen (Vorsitz); Dr. med. Felix Brem, Weinfelden; Pascal Diacon, Pflege, Zürich; Gerhard Grossglauser, Bolligen; Dr. med. Danielle Gubser, Neuenburg; lic. phil. Ruedi Haltiner, Chur; lic. phil. Heidi Lauper, Bern; Dr. med. Mark Mäder, Basel; Dr. med. Valdo Pezzoli, Lugano; Dr. med. Judit Lilla Pók Lundquist, Zürich; Prof. Dr. med. Claude Regamey, Präsident ZEK, Freiburg; lic. iur. Michelle Salathé, SAMW, Basel

Beigezogene Experten: Dr. med. Dick Joyce, Allschwil; Dr. med. Jackie Leach Scully, Basel; Claudine Braissant, Pflege, Belmont; PD Dr. med. Barbara Jeltsch-Schudel, Winterthur; Dr. med. Roland Kunz, Affoltern a.A.; Dr. theol. Markus Zimmermann-Acklin, Luzern

Vernehmlassung: Am 29. November 2007 hat der Senat der SAMW eine erste Fassung dieser Richtlinien zur Vernehmlassung genehmigt.

Genehmigung: Die definitive Fassung dieser Richtlinien wurde am 20. Mai 2008 vom Senat der SAMW genehmigt.
}

\section{Sozial-medizinische Institution}

Als «sozial-medizinische Institution» werden Institutionen bezeichnet, in denen Menschen mit Behinderung neben anderen Leistungen professionelle Pflege erhalten.

\section{Hinweise}

1 Murray CJL, Lopez AD. Quantifying disability: data, methods and results. Bulletin of the World Health Organisation. 1994;72(3):481-94 (http://whqlibdoc.who.int/bulletin/1994/ Vol72-No3/bulletin_1994_72(3)_481-494pdf).

2 Vgl. zur Definition von «Behinderung» Kap. 1 und Definition im Glossar.

3 Vgl. die am 13. Dezember 2006 von der Generalversammlung der Vereinten Nationen zur Ratifikation freigegebene «Menschenrechtskonvention über die Rechte von Personen mit Behinderungen» (www.un.org/disabilities/convention/conventionfull.shtml) sowie das Bundesgesetz vom 13. Dezember 2002 über die Beseitigung von Benachteiligung von Menschen mit Behinderungen (BehiG).

4 Der Begriff «medizinisch» wird nachfolgend umfassend verwendet und bezieht sich auf die Tätigkeit von Ärzten, Pflegenden und Therapeuten.

5 Die entsprechenden Texte betreffen nachfolgend immer auch die weiblichen Angehörigen einer Personengruppe.

6 Mit Aufnahme in die Standesordnung FMH werden die Richtlinien für FMH-Mitglieder verbindliches Standesrecht.

7 Die Richtlinien wenden sich primär an Fachpersonen der Medizin, sie sprechen aber auch Fachpersonen im Bereich Sozialpädagogik, Agogik usw. an.

8 Zur Definition von «sozialer Institution» vgl. Glossar.

9 Zur Definition von «Gesundheitsproblem» vgl. Glossar.

10 Dies umfasst sowohl Störungen motorischer und sensorischer Körperfunktionen wie auch Beeinträchtigungen kognitiver und anderer psychischer Funktionen.

11 Zur Herkunft der Definition von «Behinderung» vgl. Glossar.

12 Zum Konzept der «Menschenwürde» vgl. Glossar.

13 Immer wenn von Menschen mit Behinderung als Empfängern von medizinischen Leistungen die Rede ist, wird bewusst von Patienten und nicht allgemeiner von Personen oder Menschen gesprochen, um der primär medizinischen Bedeutung dieser Richtlinien gerecht zu werden.

14 Als «Angehörige» werden der Lebenspartner und die nächsten Verwandten eines Patienten sowie ihm nahestehende Personen bezeichnet.

15 Vgl. Glossar unter «Behinderung»

16 Mit persönlicher Assistenz werden alle Formen der Unterstützung bezeichnet, bei denen die Betroffenen selbst bestimmen, wer ihnen wo, wann und wie behilflich ist. Die erbrachten Leistungen werden in der Regel durch den Auftraggeber selber abgegolten.

17 Vgl. hierzu insb. «Recht der Patientinnen und Patienten auf Selbstbestimmung», Medizinischethische Grundsätze der SAMW. 
18 Vgl. Kap. 3 Kommunikation.

19 Urteilsfähigkeit bedeutet die Fähigkeit, die Realität wahrzunehmen, sich Urteil und Wille zu bilden, sowie die Fähigkeit, die eigene Wahl zu äussern.

Vgl. weiter die ausführliche Begriffserläuterung in: «Recht der Patientinnen und Patienten auf Selbstbestimmung», Medizinisch-ethische Grundsätze der SAMW.

20 Vgl. aber nachfolgend Kap. 7.

21 Starke Emotionen wie z.B. Ängste können das Vorhandensein der Urteilsfähigkeit in Frage stellen, so dass nicht mehr von einer autonomen Willensäusserung gesprochen werden kann.

22 Über das Vorgehen bei urteilsunfähigen Patienten bestehen in verschiedenen Kantonen explizite gesetzliche Bestimmungen, die Vorrang vor den Richtlinien haben.

23 Es handelt sich hier um die sogenannte genuine bzw. ursprüngliche Urteilsunfähigkeit bei Patienten, die seit Geburt nicht fähig waren, einen eingriffsrelevanten Willen zu bilden (z.B. Menschen mit einer schweren geistigen Behinderung).

24 Gesetzliche Vertreter sind Personen, die aufgrund einer ihnen eingeräumten gesetzlichen Befugnis den Patienten vertreten.

25 Aufgrund der geltenden Bestimmungen auf Bundesebene haben Angehörige keine Vertretungsbefugnis. In einigen Kantonen sind jedoch dem urteilsunfähigen volljährigen Patienten nahestehende Personen oder ein Familienmitglied gesetzlich legitimiert, die Einwilligung zu einer Behandlung zu erteilen.

26 Zur Lebensrettung oder zur Abwehr schwerer Folgeschädigung darf eine unaufschiebbare Behandlung, Pflege oder Betreuung durchgeführt werden, wenn weder der Patient selbst noch sein Vertreter rechtzeitig einwilligen kann oder wenn begründete Zweifel an der Gültigkeit einer Behandlungsverweigerung wegen Urteilsunfähigkeit oder Interessenkollision beim gesetzlichen Vertreter bestehen.

27 Vgl. Kap. 6 Dokumentation.

28 Vgl. «Palliative Care», Medizinisch-ethische Richtlinien der SAMW.

29 Vgl. «Betreuung von Patientinnen und Patienten am Lebensende», Medizinisch-ethische Richtlinien der SAMW

30 Je nach der Rechtsnatur des Behandlungsverhältnisses beurteilt sich die Frage der Dokumentationspflicht nach den Normen des Bundesprivatrechts (OR) oder des kantonalen öffentlichen Rechts.

31 Für Ärzte und Medizinalpersonen gilt das Berufsgeheimnis gemäss Art. 321 StGB; für Personal einer Institution, das nicht unter die Bestimmungen von Art. 321 fällt, gelten arbeitsvertragliche Regelungen in bezug auf die Schweigepflicht; teilweise ist die Schweigepflicht auch im kantonalen Recht geregelt.

32 Vgl. Art. 321 StGB Ziff. 2 und 3.

33 Vgl. «Zwangsmassnahmen in der Medizin», Medizinisch-ethische Richtlinien der SAMW.

34 Als Zwangsmassnahmen werden alle Eingriffe bezeichnet, die gegen den erklärten Willen oder gegen Widerstand eines Menschen - oder bei Kommunikationsunfähigkeit gegen den mutmasslichen Willen - erfolgen. Darunter fallen sowohl freiheitsbeschränkende Massnahmen als auch Zwangsbehandlung.
$35 \mathrm{Zu}$ den rechtlichen Rahmenbedingungen vgl. ausführlich die Richtlinien «Zwangsmassnahmen in der Medizin», Kapitel 3.2.1.

36 Zur Evaluation gehört auch eine fortlaufende Dokumentation.

37 Delikte gegen Leib und Leben.

38 Maslow Abraham H. Motivation und Persönlichkeit. Reinbek: Rowohlt; 1991.

39 Kantonale Gesundheitsgesetze sehen eine Meldepflicht, teilweise ein Melderecht für Ärzte vor, be Verdacht auf Delikte gegen Leib und Leben oder die sexuelle Integrität eine polizeiliche Anzeige zu erstatten, ohne dass eine Entbindung von der Schweigepflicht vorliegt.

40 Bundesgesetz über Voraussetzungen und Verfahren bei Sterilisationen vom 17. Dezember 2004

41 Der unvermittelte Übergang zur Höflichkeitsform ab einem bestimmten Alter kann ebenso irritierend sein wie unreflektiertes Duzen bei Erwachsenen.

42 Für Versicherte vor dem vollendeten 20. Alterjahr übernimmt die IV die Kosten für alle zur Behandlung eines anerkannten Geburtsgebrechens notwendigen medizinischen Massnahmen. Die obligatorische Krankenpflegeversicherung übernimmt subsidiär die Kosten von Behandlungen von Geburtsgebrechen, die von der IV nicht übernommen werden.

Bei Erwachsenen nach dem 20. Altersjahr werden die Behandlungskosten von Krankheiten oder Unfallfolgen von der Kranken- bzw. Unfallversicherung übernommen. Die IV übernimmt nur noch die Kosten für medizinische Massnahmen, die unmittelbar die berufliche Eingliederung fördern und geeignet sind, die Erwerbsfähigkeit dauernd und wesentlich zu verbessern oder wesentliche Beeinträchtigungen der Erwerbsfähigkeit zu verhindern. In diesem Rahmen kann die IV die Kosten übernehmen für die ärztliche Behandlung (ambulant oder in der allgemeinen Abteilung eines Spitals), die Behandlung durch medizinische Hilfspersonen (Physiotherapeuten usw.) und für anerkannte Arzneimittel. (Vgl. Bundesgesetz über die Invalidenversicherung vom 19. Juni 1959 und Ausführungsverordnungen sowie Bundesgesetz über die Krankenversicherung vom 18. März 1994.)

43 So sind beispielsweise Kinderspitäler zum Teil bezüglich Aufnahme von Patienten an die Altersgrenze von 16 Jahren gebunden, obwohl in vielen Fällen eine Weiterbetreuung und ein stufenweiser Übergang in die Erwachsenenmedizin sinnvoll sind.

44 Vgl. «Behandlung und Betreuung von älteren, pflegebedürftigen Menschen», Medizinisch-ethische Richtlinien und Empfehlungen der SAMW.

45 Der Anspruch auf IV-Renten und auf Übernahme der Kosten für medizinische Massnahmen zur beruflichen Eingliederung erlischt, wenn die Voraussetzungen nicht mehr erfüllt sind, spätestens aber, wenn der IV-Rentner oder die IV-Rentnerin das AHV-Alter erreicht bzw. Anspruch auf eine Altersrente hat.

46 Vgl. auch Art. 17 des Übereinkommens über Menschenrechte und Biomedizin vom 4. April 1997, das von der Schweiz ratifiziert wurde. 